\title{
Robust neuroinflammation and perivascular pathology in rTg-DI rats, a novel model of microvascular cerebral amyloid angiopathy
}

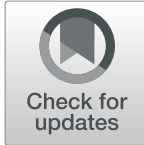

Xiaoyue Zhu', Joshua Hatfield ${ }^{1,2}$, Joseph K. Sullivan ${ }^{1,3}$, Feng Xu' and William E. Van Nostrand ${ }^{1 *}$ (D)

\begin{abstract}
Background: Cerebral amyloid angiopathy (CAA) is a common cerebral small vessel disease of the aged and a prominent comorbidity of Alzheimer's disease (AD). CAA can promote a variety of vascular-related pathologies including neuroinflammation, cerebral infarction, and hemorrhages, which can all contribute to vascular cognitive impairment and dementia (VCID). Our understanding of the pathogenesis of CAA remains limited and further investigation of this condition requires better preclinical animal models that more accurately reflect the human disease. Recently, we generated a novel transgenic rat model for CAA (rTg-DI) that develops robust and progressive microvascular CAA, consistent microhemorrhages and behavioral deficits.
\end{abstract}

Methods: In the current study, we investigated perivascular pathological processes that accompany the onset and progressive accumulation of microvascular CAA in this model. Cohorts of rTg-DI rats were aged to 3 months with the onset of CAA and to 12 months with advanced stage disease and then quantitatively analyzed for progression of CAA, perivascular glial activation, inflammatory markers, and perivascular stress.

Results: The rTg-DI rats developed early-onset and robust accumulation of microvascular amyloid. As the disease progressed, rTg-DI rats exhibited increased numbers of astrocytes and activated microglia which were accompanied by expression of a distinct subset of inflammatory markers, perivascular pericyte degeneration, astrocytic caspase 3 activation, and disruption of neuronal axonal integrity.

Conclusions: Taken together, these results demonstrate that rTg-DI rats faithfully mimic numerous aspects of human microvascular CAA and provide new experimental insight into the pathogenesis of neuroinflammation and perivascular stress associated with the onset and progression of this condition, suggesting new potential therapeutic targets for this condition. The rTg-DI rats provide an improved preclinical platform for developing new biomarkers and testing therapeutic strategies for microvascular CAA.

Keywords: Cerebral amyloid angiopathy, Alzheimer's disease, Transgenic rats, Beta amyloid, Neuroinflammation, Perivascular stress

\footnotetext{
* Correspondence: wvannostrand@uri.edu

'Department of Biomedical and Pharmaceutical Sciences, College of

Pharmacy, George \& Anne Ryan Institute for Neuroscience, University of

Rhode Island, 130 Flagg Road, Kingston, RI 02881, USA

Full list of author information is available at the end of the article
}

(c) The Author(s). 2020 Open Access This article is licensed under a Creative Commons Attribution 4.0 International License, which permits use, sharing, adaptation, distribution and reproduction in any medium or format, as long as you give appropriate credit to the original author(s) and the source, provide a link to the Creative Commons licence, and indicate if changes were made. The images or other third party material in this article are included in the article's Creative Commons licence, unless indicated otherwise in a credit line to the material. If material is not included in the article's Creative Commons licence and your intended use is not permitted by statutory regulation or exceeds the permitted use, you will need to obtain permission directly from the copyright holder. To view a copy of this licence, visit http://creativecommons.org/licenses/by/4.0/. The Creative Commons Public Domain Dedication waiver (http://creativecommons.org/publicdomain/zero/1.0/) applies to the data made available in this article, unless otherwise stated in a credit line to the data. 


\section{Background}

Cerebral amyloid angiopathy (CAA) is a prominent cerebral small vessel disease characterized by the deposition of fibrillar amyloid beta peptide (A $\beta)$ within small arteries and arterioles of meninges and cortex as well as the brain capillaries $[1,2]$. Sporadic CAA is common in the elderly brain and is present in $>50 \%$ of individuals over the age of 80 years [3]. It has been reported that greater than $80 \%$ Alzheimer's disease (AD) patients present CAA pathology in varying levels [1-4]. Further, early-onset, familial forms of CAA result from specific mutations within the $A \beta$ peptide including the Dutch E22Q and Iowa D23N variants [5-7]. Clinically, CAA can promote cerebral infarction, intracerebral hemorrhages $(\mathrm{ICH})$, and microbleeds $[1,2,8$, 9]; all of which can contribute to vascular cognitive impairment and dementia (VCID).

There are two prominent forms of CAA. When restricted to the meningeal and intracortical cerebral arterioles, this is referred as CAA type- $2[1,10]$. In CAA type- 1 , the deposition of $A \beta$ is present on the capillary walls and observed in approximately half of AD cases [10]. In contrast to larger-vessel CAA type-2, CAA type-1 results in fibrillar amyloid penetrating the surrounding brain parenchyma, referred to as dyshorric amyloid, which further promotes a strong perivascular neuroinflammatory response $[1,10-12]$. It is suggested that the early pericapillary $A \beta$ impairs the perivascular drainage pathway, which leads to the disruption of $A \beta$ clearance resulting in more $A \beta$ deposition on capillary walls [1]. Further, CAA type- 1 is often correlated with impaired cognition and rapidly progressive dementia [13-15]. Although CAA type-1 shows high clinical relevance, its contributions to neurodegenerative diseases is still unclear. Therefore, a better understanding of the mechanisms involved in CAA pathogenesis may be helpful in the design of therapeutic approaches targeting this condition.

Recently, we generated a novel transgenic rat model (rTg-DI) that produces low levels of human familial CAA Dutch/Iowa E22Q/D23N mutant $A \beta$ in the brain [16]. The rTg-DI rat model shows many pathologic aspects of human small vessel CAA type-1, including similar vascular $A \beta$ structure, glial activation, and microhemorrhages, accompanied by behavioral deficits [16]. In order to gain a better understanding of the mechanisms underlying CAA pathology, we investigated perivascular pathological processes that accompany CAA type- 1 in the rTg-DI rat model. Here we show that rTg-DI rats exhibit early-onset and progressive accumulation of capillary fibrillar $\mathrm{A} \beta$ paralleled with increasing numbers of perivascular glial cells. Furthermore, accumulating vascular amyloid promoted expression of a subset of inflammatory markers, which was accompanied by loss of perivascular pericytes, induction of cellular caspase 3, and axonal pathology. Collectively, this work demonstrates that the rTg-DI model faithfully recapitulates multiple aspects of human CAA type- 1 and provides further insight into emerging CAA type-1 pathologies, which may offer new targets in developing appropriate therapeutic interventions.

\section{Methods}

\section{Animals}

The generation of rTg-DI transgenic rats was recently described [16]. These rats modestly express human Swedish/Dutch/Iowa mutant A $\beta$ PP under the control of the neuronal-specific Thy1.2 promoter. The deposition of vascular fibrillar mutant Dutch/Iowa A $\beta$ peptide begins at around 3 months of age with a progressive increase in the amounts of primarily soluble and insoluble pools $\mathrm{A} \beta 40$ in the brain with age. Heterozygous rTg-DI rats and non-transgenic, wild-type rats at 3 and 12 months of age were used in the present study. All work with animals was in accordance with the United States Public Health Service's Policy on Humane Care and Use of Laboratory Animals and was approved by the University of Rhode Island Institutional Animal Care and Use Committee (IACUC).

\section{Brain tissue preparation}

Animals were euthanized with $\mathrm{CO}_{2}$ at specified ages. The rat brain was surgically removed and bisected in the mid-sagittal plane. One hemisphere was snap-frozen in liquid nitrogen for mRNA and protein analyses. The other hemisphere was either fixed in $4 \%$ paraformaldehyde or fixed in $70 \%$ ethanol followed by xylene treatment and embedding in paraffin or snap-frozen in optimal cutting temperature medium (OCT 4585, Fisher Healthcare) directly.

\section{Immunohistochemistry}

For glial cell analysis, paraffin-embedded brains were sagittally sectioned at $10 \mu \mathrm{m}$, or for stereological analysis at $50 \mu \mathrm{m}$, thickness using a microtome, deparaffinated and rehydrated. Then sections were incubated with proteinase $\mathrm{K}\left(0.2 \mathrm{mg} \mathrm{mL}^{-1}\right)$ for $5 \mathrm{~min}$ at room temperature. Sections were then blocked in Superblock blocking buffer (37518, ThermoFisher) containing 0.3\% Triton X-100 at room temperature for $30 \mathrm{~min}$ and incubated with individual primary antibodies at the following dilutions overnight: rabbit polyclonal antibody to collagen IV (1: 250, SD2365885, Invitrogen), goat polyclonal antibodies to glial fibrillary acidic protein (GFAP, 1:250, ab53554, Abcam), or ionized calcium-binding adapter molecule 1 (Iba-1, 1:250, NB100-1028, Novus). Mouse monoclonal antibody to OX6 MHCII (1:200, Abcam) was used to identify cells in the brain that potentially represent macrophagic microglia.

For the pericyte staining, the fresh-frozen OCT directly embedded tissues were used. Sections were fixed in 
acetone for $10 \mathrm{~min}$, then air dried for $30 \mathrm{~min}$ followed by PBS rehydration for $5 \mathrm{~min}$ at room temperature. Sections were blocked in Superblock blocking buffer for 30 min then incubated with goat polyclonal antibody to platelet-derived growth factor receptor beta (PDGFR $\beta, 1$ : 250 , gov0415021, R\&D) and rabbit polyclonal antibody to collagen IV overnight.

For fluorescence staining, after the overnight incubation, sections were washed with PBS for three times, then incubated with Alexa Fluorescent 594- or 488-conjugated secondary antibodies (1:1000). Fluorescent staining for fibrillar amyloid was performed using either thioflavin $S$ (123H0598, Sigma-Aldrich) or Amylo-Glo (TR-300-AG, Biosensis Inc.), as described by the manufacturer. Nuclear staining was performed with 4',6-diamidino-2-phenylindole (DAPI, 10236276001, Sigma-Aldrich).

\section{Quantitative measures of CAA pathologies}

The percent area of capillaries covered with amyloid was quantified using a set of sections that were stained with thioflavin $\mathrm{S}$ and immunolabeled with antibody to collagen IV. A series of non-overlapping images covering the cortex, hippocampus, and thalamus were captured and analyzed by the Image J software. For each field, the sum area of thioflavin $\mathrm{S}$ was divided by that for the total collagen IV immunoreactive capillary area $\times 100$ to yield the percent area of microvascular amyloid.

The numbers of astrocytes and microglia, and pericytes in capillaries of the cortex, hippocampus, and thalamus at each 3 and 12 months of age were determined using stereological principles [17]. The density of microglia and astrocytes was quantified in the regions of the cortex, thalamus, and hippocampus of rTg-DI and agematched WT rats. The total numbers of microglia and astrocytes were estimated using the Stereologer software system (Systems Planning and Analysis). Every tenth section cut at $50 \mu \mathrm{m}$ was selected and generated 10-15 sections per reference space in a systematic-random manner. Immunopositive cells were counted using the optical fractionator method with the dissector principle and unbiased counting rules [17]. Criteria for counting cells required that cell bodies exhibited positive GFAP or Iba-1 immunostaining, for astrocytes or microglia respectively.

The density of pericytes was calculated using a set of brain sections labeled with antibodies to PDGFR $\beta$ for pericytes and collagen IV to identify capillaries/microvessels and Amylo-Glo to reveal deposited fibrillar amyloid. The number of pericyte cell bodies and the length of capillaries were calculated in the BZ-X Analyzer software. Then, the pericyte coverage in each area was calculated by the total number of pericytes divided the sum length of capillaries.

\section{Real-time quantitative PCR}

Rat brains at each age were collected and snap-frozen in liquid nitrogen. The brains were first homogenized within Trizol (162711, Invitrogen), then the total RNA was extracted by using the Direct-zol RNA MiniPrep kit (ZRC200796, ZYMO research) according to the manufacturer's protocol. Total RNA concentrations were measured using Nanodrop (Nanodrop one, Thermo Scientific). cDNA was synthesized with the High Capacity cDNA Reverse Transcription Kit (00289994, Applied Biosystems) following manufacturer's guidelines. Quantitative PCR (qPCR) were performed in the Step One Plus Real-time PCR system (Applied Biosystems) using the Taqman primers (CD86, Rn005 71654_m1; CD68, TREM2, Rn01512170_m1; Rn01495634_ g1; GFAP, Rn00566603_m1; TGFß1, Rn00572010_m1; TNFo, Rn01525859_g1; IL1 $\beta, \quad$ Rn00580432_m1; IL10, Rn01483988_g1; IL6, Rn01410330_m1; IL17, Rn01757168_ m1; C3, Rn00566466_m1; C1q,Rn00595250_m1; C4b, Rn 01774112_mH; C1inh, Rn01485600_m1; MMP9, Rn0057 9162_m1; MMP2, Rn01538170_m1; Actb, Rn00667869_ $\mathrm{m} 1)$. Obtained mRNA expression levels were normalized to Actin-beta.

\section{Identification of pro-apoptotic cells}

The numbers of pro-apoptotic cells were determined using stereological principles as described above. Sections of 3-month and 12-month wild-type and rTg-DI rat brains were immunolabeled with a rabbit antibody for active-caspase 3 for identifying the pro-apoptotic cells (1:250, AF835, Novus), mouse monoclonal antibody 66. 1[18] (1:250) to label fibrillar amyloid and stained with DAPI for nuclear labeling for counting the total number of cells. The ratio of caspase 3-positive cells to total cell number in cortex, hippocampus, and thalamus were calculated using the BZ-X Analyzer software. To identify the caspase 3-positive cell types, the brain sections were double immunolabeled with antibody to caspase 3 and with antibodies to NeuN, GFAP, or Iba- 1 to identify neurons, astrocytes, or microglia, respectively.

\section{Analysis of axonal pathology}

Axonal integrity was evaluated by immunolabeling the brain sections from 3 and 12-month-old wild-type and rTg-DI rats with SMI312 mouse monoclonal pan axonal neurofilament marker (1:200, 837904, Biolegend). Tissue sections were stained with thioflavin $\mathrm{S}$ to identify microvascular fibrillar amyloid deposits.

\section{Statistical analysis}

Statistical analysis was performed using the Graphpad Prism software. Results are shown as mean value with standard deviation (SD) of the mean. The statistical differences between pairs of data sets were analyzed by $t$ test at the 0.05 significance level. 


\section{Results}

Progressive accumulation of microvascular amyloid is accompanied by increased numbers and activation of glial cells

rTg-DI rats exhibit progressive accumulation of cerebral microvascular fibrillar amyloid in the cortex, hippocampus, and thalamus (Fig. 1). The deposition of microvascular amyloid begins at $\approx 3$ months of age in all three brain regions (Fig. 1a-c). At 12 months of age, the rTg-DI rats developed extensive cerebral microvascular $A \beta$ deposition, with more than $30 \%$ and $60 \%$ of the capillaries covered by fibrillar $A \beta$ in the hippocampus and thalamus, respectively (Fig. 1g). Although the level of CAA was somewhat lower in the cortical area, still more than $15 \%$ of the vessel surface was covered with amyloid. These findings demonstrate that the rTg-DI rat model consistently develops early-onset and extensive cerebral vascular $\mathrm{A} \beta$ accumulation in the brain. For all of the proceeding analyses, measures were performed at the early-stage onset of microvascular CAA (3 months) and compared with late-stage disease with extensive microvascular CAA (12 months).
Astrocytes have been increasingly recognized as an important contributor to the neuroinflammatory and neurodegenerative processes in $\mathrm{A} \beta$-related human disorders including CAA [19]. The accumulation of cerebral microvascular amyloid in $\mathrm{rTg}$-DI rats similarly promotes a strong astroglial response in several ways. For example, at 3 months of age with the onset of microvascular amyloid deposition, there is a noticeable uptick in the numbers of astrocytes and the beginning of a change in appearance compared to age-matched wild-type rats (Fig. 2a-f, o). These early changes observed in 3 months old rTg-DI rats were not evident in younger 1 month old animals, prior to onset of microvascular amyloid deposition (Additional Fig. 1). As rTg-DI rats aged to 12 months and presented with extensive microvascular CAA (Fig. 1), there was a dramatic increase in the numbers of astrocytes in the cortical, hippocampal, and thalamic regions (Fig. $2 \mathrm{j}-\mathrm{l}, \mathrm{o}$ ) whereas the numbers of astrocytes in these brain regions of wild-type rats remained fairly constant compared to 3 months of age (Fig. 2g-i, o). Further, a pronounced morphological alteration of astrocytes was observed in rTg-DI rats. In contrast

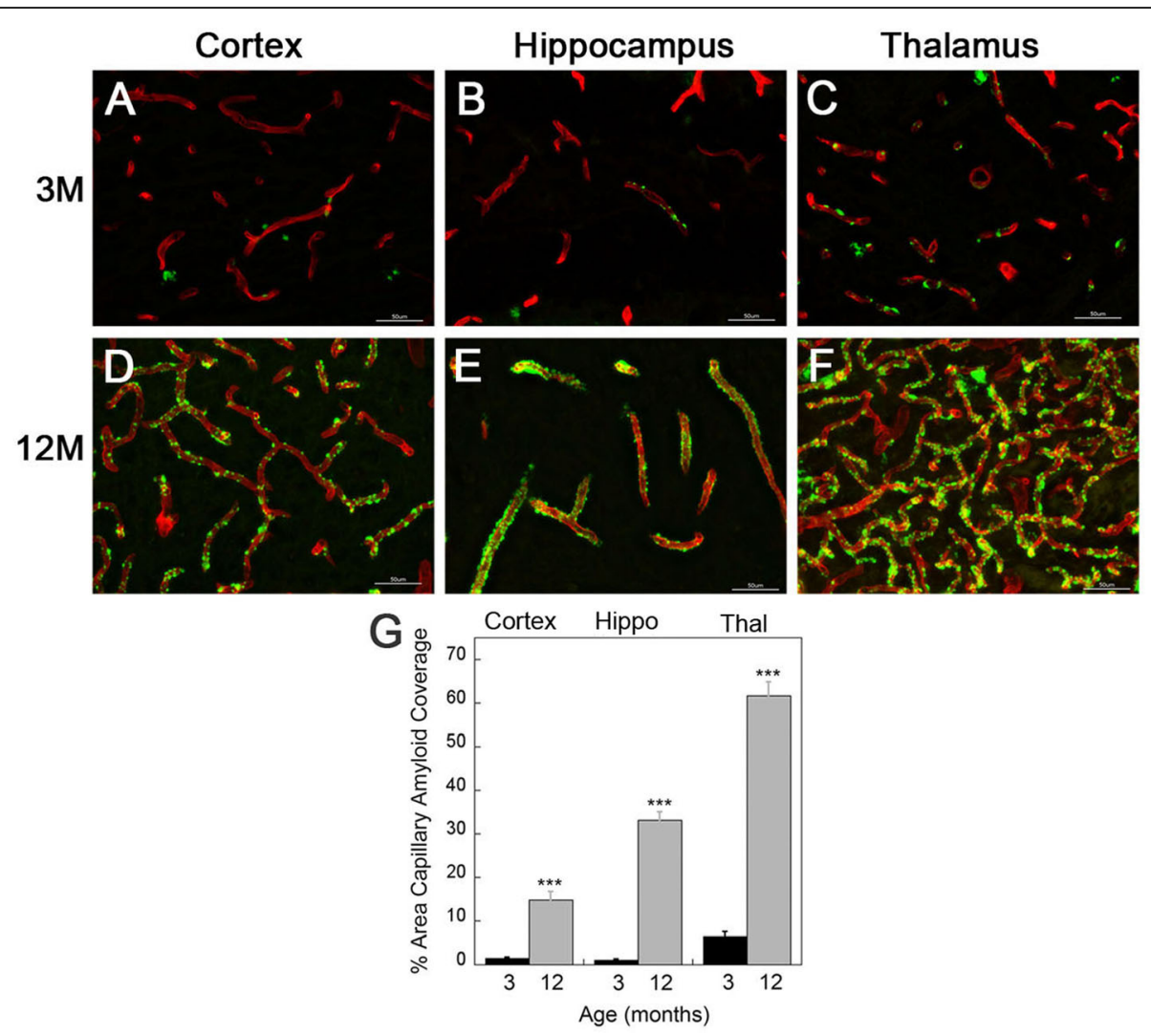

Fig. 1 Quantitative analysis of progressive accumulation of microvascular CAA in rTg-DI rats. a-f Brain sections from 3-month-old (a-c) and 12month-old ( $\mathbf{d}-\mathbf{f}$ ) $\mathrm{rTg}$-DI rats were immunolabeled with rabbit polyclonal antibody to collagen IV to specifically detect cerebral microvessels (red) and the thioflavin $\mathrm{S}$ to identify fibrillar amyloid (green). The $\mathbf{r T g}$-DI rats showed progressive cerebral microvascular fibrillar amyloid deposition in the cortical (a, d), hippocampal (b, e), and thalamic regions (c, f). Scale bars $=50 \mu \mathrm{m}$. $\mathbf{g}$ Quantitation of cerebral microvascular amyloid load in different brain regions of 3-month-old (black bars) and 12-month-old (gray bars) rTg-DI rats. Data are expressed as means \pm SD of $n=6-7$ rTg-DI rats per group. ${ }^{* *} P<0.001$ 

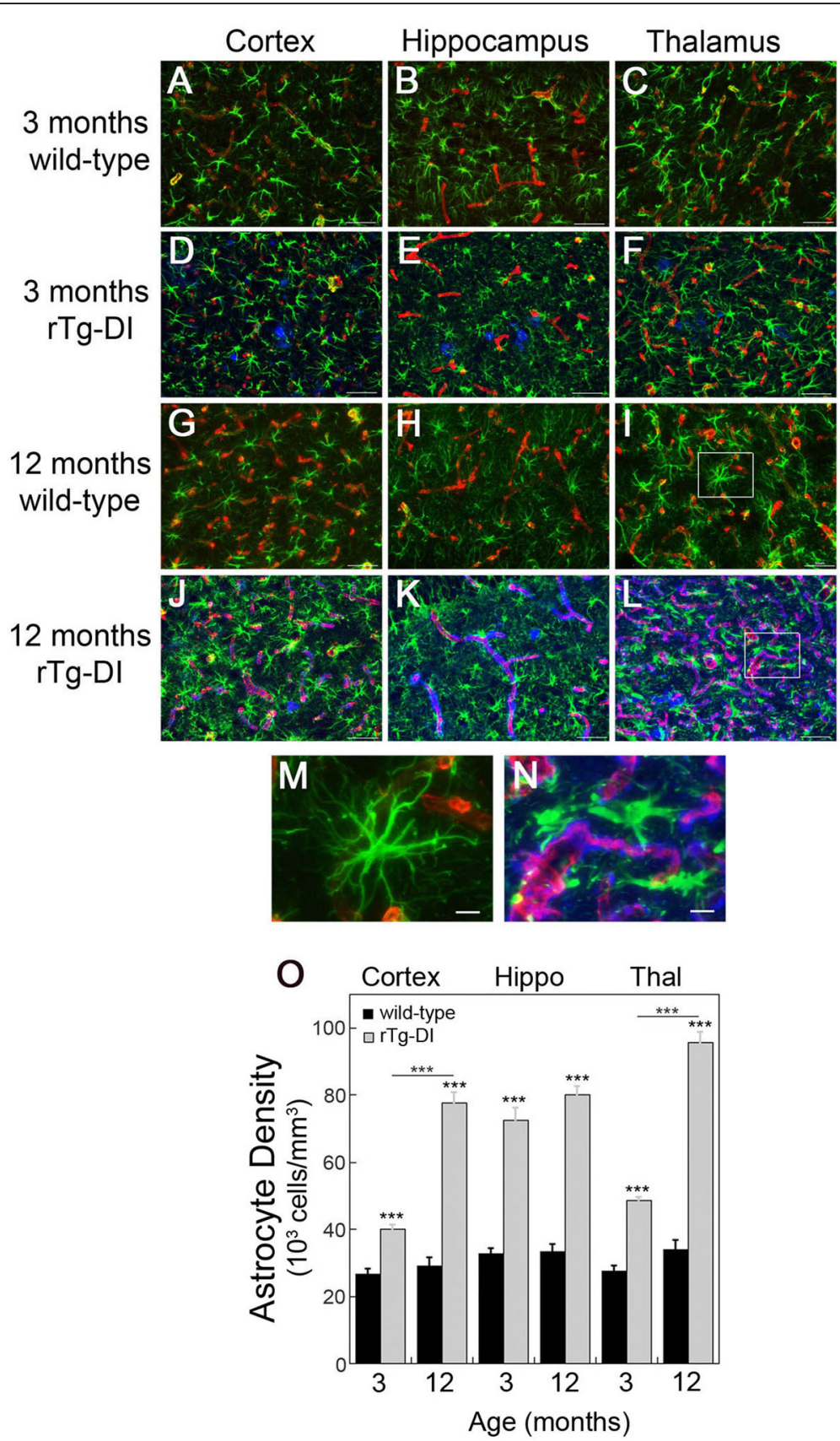

Fig. 2 Increased perivascular astrocytes in rTg-DI rats. a-I Brain sections from 3-month-old wild-type (a-c) and rTg-DI (d-f) rats and 12-month wildtype ( $\mathbf{g}-\mathbf{i})$ and $\mathbf{r T g}-\mathrm{DI}(\mathbf{j}-\mathbf{I})$ rats were labeled with Amylo-Glo to detect fibrillar amyloid (blue), rabbit polyclonal antibody to collagen IV to detect cerebral microvessels (red), and goat polyclonal antibody to GFAP to identify astrocytes (green). Scale bars $=50 \mu m$. $\mathbf{m}, \mathbf{n}$ Enlarged images of the highlighted regions of panels $\mathbf{i}$ and $\mathbf{I}$, respectively. Scale bars $=10 \mu \mathrm{m}$. $\mathbf{0}$ Quantitation of astrocyte numbers from wild-type rats (black bars) and rTg-DI rats (gray bars) in different brain regions at 3 and 12 months of age. Data shown are mean \pm SD of $n=5-6$ rats per group. Compared to wild-type rats the astrocyte numbers were markedly elevated in rTg-DI rats and increased from 3 to 12 months of age in measured brain regions. ${ }^{* *} P<0.001$

to the astrocytes present in the brains of 12-month wildtype rats (Fig. $2 \mathrm{~m}$ ), the astrocytes surrounding microvessels with amyloid generally showed increased cell body volume and thickened, retracted processes (Fig. 2n).

Activated microglia have also been strongly associated with human CAA type-1 vascular amyloid since it engages the surrounding brain parenchyma [12, 20, 21]. We found that in rTg-DI rats the presence of microvascular amyloid dramatically changes the numbers and morphological state of microglia. At 3 months of age, with onset of microvascular amyloid, the numbers of microglia sharply rose compared to wild-type rats (Fig. 3a-f, o). As the rTg-DI rats 
further aged to 12 months, presenting with more extensive microvascular amyloid, the numbers of microglia further increased in all brain regions whereas they remained constant in wild-type rats (Fig. $3 g-1$, o). In addition to these marked increases in numbers, the microglia surrounding the microvascular amyloid deposits showed a pronounced change in morphology. In 12-month-old wild-type rats, microglia are sparse and exhibit a resting, surveillance phenotype with long extended process (Fig. $3 \mathrm{~m}$ ). In contrast, the presence of microvascular amyloid promotes a distinct change in morphology to activated state with enlarged cell bodies and retracted processes that are closely engaged with the

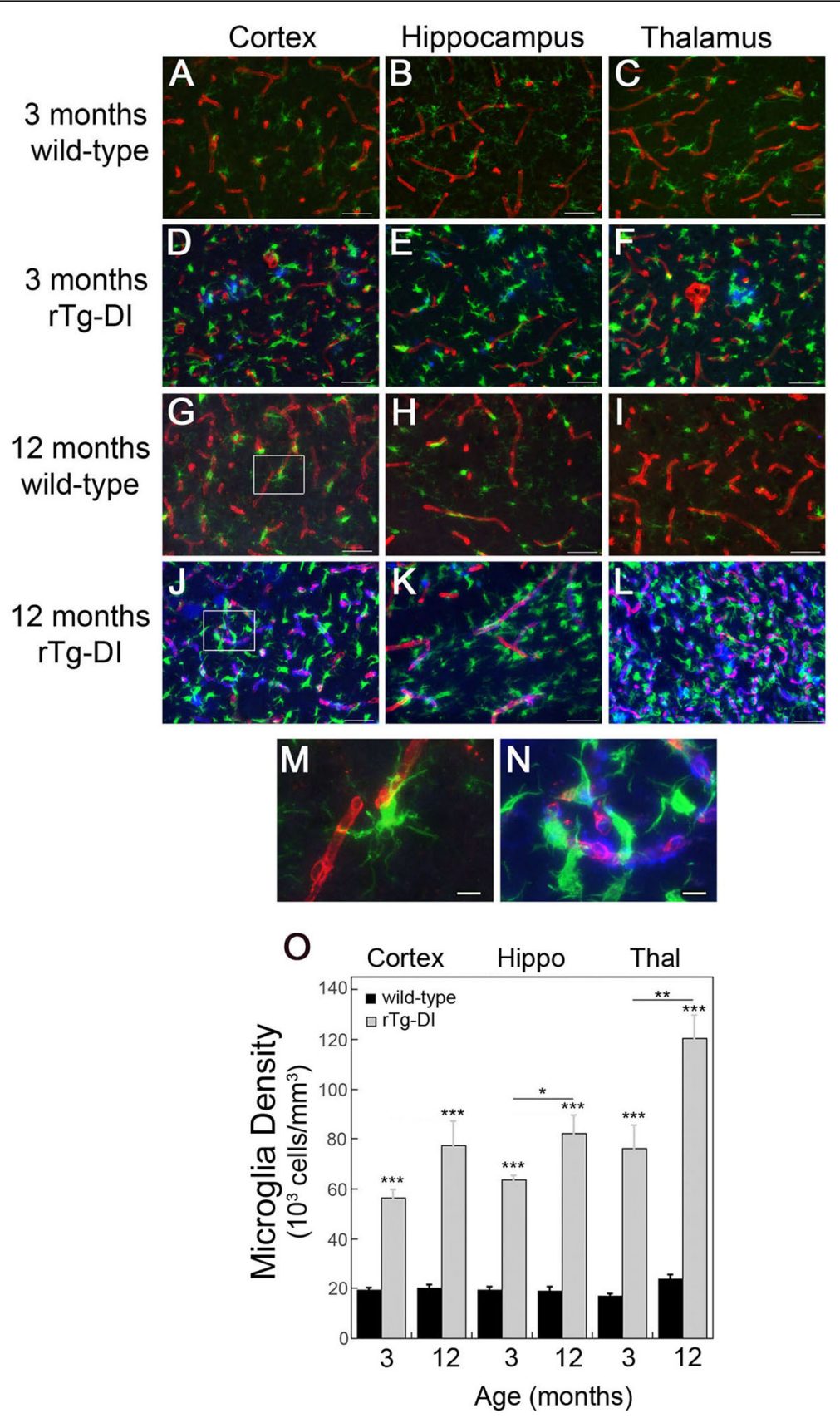

Fig. 3 Increased perivascular microglia in rTg-DI rats. a-I Brain sections from 3-month-old wild-type (a-c) and rTg-DI (d-f) rats and 12-month wild-type (g-i) and $\mathbf{r T g}-\mathrm{DI}(\mathbf{j}-\mathbf{I})$ rats were labeled with Amylo-Glo to detect fibrillar amyloid (blue), rabbit polyclonal antibody to collagen IV to detect cerebral microvessels (red), and goat polyclonal antibody to lba-1 to identify microglia (green). Scale bars $=50 \mu \mathrm{m} . \mathbf{m}, \mathbf{n}$ Enlarged images of the highlighted regions of panels $\mathbf{g}$ and $\mathbf{j}$, respectively. Scale bars $=10 \mu \mathrm{m}$. o Quantitation of microglia numbers from wild-type rats (black bars) and rTg-DI rats (gray bars) in different brain regions at 3 and 12 months of age. Data shown are mean \pm SD of $n=5-6$ rats per group. Compared to wild-type rats the microglia numbers were markedly elevated in rTg-Dl rats and increased from 3 to 12 months of age in the measured brain regions. ${ }^{*} P<0.02,{ }^{* *} P<0.01,{ }^{* * *} P<0.001$ 
amyloid (Fig. 3n). These changes in microglia numbers and morphology were not evident in 1-month-old rTg-DI rats prior to microvascular amyloid deposition (Additional Fig. 2 ). At this young age, microglia were indistinguishable between wild-type and rTg-DI rats with both exhibiting a resting state with elaborate extended processes.

To investigate potentially different subtypes of microglia in rTg-DI rats, we performed double immunolabeling for Iba-1, a common resident microglial marker, and OX6 MHCII a marker that has been used to identify activated macrophagic microglia [22, 23]. In 3 and 12-month wildtype rats, the small number of microglia were labeled exclusively with Iba-1 and exhibited their resting state morphology (Additional Fig. 3). On the other hand, in the presence of microvascular amyloid at 3 months and more so at 12 months, a subset of microglia was labeled with both Iba-1 and OX6. However, there were few, if any, cells labeled solely with OX6. This suggests that a subpopulation of microglia with a potentially different activation state exists in rTg-DI rats, especially with advanced microvascular amyloid deposition. These series of data together show that robust increases in both astrocytes and microglia, presenting with perhaps different activation states, develop as CAA emerges and progresses in rTg-DI rats.

\section{Inflammatory marker expression in $\mathrm{rTg}$-DI rat brain}

Previous data from both human and transgenic mouse models of CAA have identified changes in expression of a number of inflammatory markers $[1,11,12,24-26]$. Based on the inflammatory cell increases described above in rTg-DI rats, analysis of inflammatory marker expression could provide further insight into the pathogenesis of CAA in this model. Therefore, we next performed qRTPCR experiments to measure the expression of a series of inflammatory markers in the rTg-DI rat model of CAA at the early-stage disease onset (3 months) and at a stage of more advanced pathology (12 months).

Since we observed significantly increased numbers of astrocytes and activated microglia in rTg-DI rat brains, as shown above in Figs. 2 and 3, we first focused on a set of cell type specific markers. Indeed, compared to wild-type rats, the rTg-DI rats exhibited significantly increased expression of GFAP at 3 months of age that further increased at 12 months of age (Fig. 4a). Similarly, we measured the activation markers cluster of differentiation 68 (CD68) and CD86 [27], as well as triggering receptor expressed on myeloid cells 2 (TREM2), and found all three genes were significantly elevated in rTg-DI rat brains at 3 months with the onset of CAA with further increases

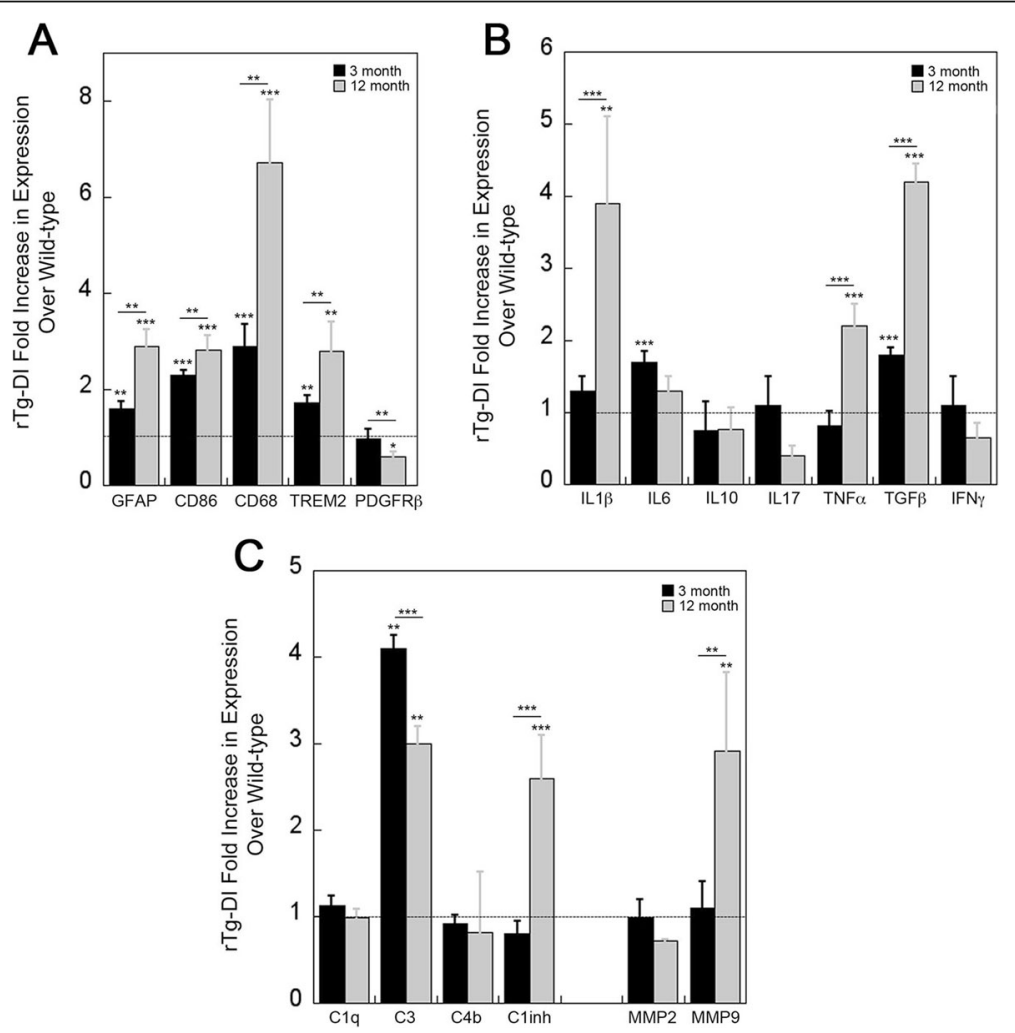

Fig. 4 Inflammation-related gene expression in rTg-DI rat brains. Total RNA was extracted from 3 and 12 months old rTg-DI rats and wild-type rats followed by reverse transcription and real-time PCR analysis of marker gene expression was performed using $\beta$-actin as an internal control. Gene expressions in wild-type animals were normalized to 1 (dash line). Data presented are the means \pm SD of $n=3-5$ rats per group. ${ }^{*} P<0.05$, ${ }^{* *} P<0.01,{ }^{* * *} P<0.001$ 
at 12 months with more advanced stage of microvascular amyloid (Fig. 4a). These findings of astrocyte and microglial marker gene expression is highly consistent with the increases in cell numbers and activation as shown above. Another cell marker measured was expression of plateletderived growth factor receptor $\beta$ (PDGFR $\beta$ ), a marker for perivascular capillary pericytes which resides in the location vascular amyloid deposition in rTg-DI rats. With the emergence of CAA at 3 months in this model, there was no difference in PDGFR $\beta$ expression compared to wildtype rats (Fig. 4a). However, as vascular amyloid accumulation became more severe at 12 months there was a significant reduction in expression of this pericyte marker.

In previous studies, CD86 expression indicated classically activated, pro-inflammatory M1 microglia [28]. Therefore, we examined the mRNA levels of IL-1 $\beta$, IL- 6 , and TNF- $\alpha$ in $r T g-D I$ rat brains, which all participate in the polarization of M1 microglia [29]. Of these markers, only IL-6 showed a significant increase in expression at the onset of CAA, but this increase subsided as the rats presented with more advanced disease at 12 months (Fig. $4 \mathrm{~b}$ ). On the other hand, increased expression of IL-1 $\beta$ and TNF- $\alpha$ was not evident at early-stage disease but was significantly elevated at late-stage disease (Fig. 4b). Interestingly, expression of IL-17, which can upregulate microglial production of IL-1 $\beta$ and IL-6 [30], was not affected or appeared to be reduced in rTg-DI rats compared to wildtype rats. We also measured the expression of two important anti-inflammatory cytokines, IL-10 and transforming growth factor $\beta$ (TGF- $\beta$ ). In this case, TGF- $\beta$ showed a significant increase in expression at 3 months with a further increase at 12 months, while IL-10 showed somewhat lower expression in $\mathrm{rTg}$-DI rats.

We next examined the expression of several complement cascade components and matrix-metalloproteinases (MMPs), which both can promote damage to cerebral blood vessels. For example, fibrillar $A \beta$ deposits have been shown to increase expression and activate certain components of the complement cascade [31]. Interestingly, the expression levels of classical complement component 1q (C1q) and C4b did not show any significant difference between rTg-DI and wild-type rats at either stage of disease. On the other hand, the alternative pathway complement component 3 (C3) showed a robust increase in expression at onset of CAA accumulation that somewhat tapered at 12 months but was still highly significant. Expression of C1-inhibitor, which regulates complement activation, was significantly elevated in advanced disease, but not with the onset of microvascular CAA. Similarly, we found disparate expression of two key MMPs that can degrade vascular basement membranes and promote bleedings [32]. Whereas MMP9 expression was increased several-fold in rTg-DI rat brains at 12 months MMP2 was not. Taken together, these findings indicate that rTg-DI rats express a unique profile of inflammatory markers, complement components, and MMPs, some beginning at the onset of microvascular amyloid accumulation with more robust expression with increased pathology at 12 months, whereas others are only expressed at late stage of disease.

\section{Decreased perivascular pericytes in $\mathrm{rTg}$-DI rats}

Pericytes are vascular mural cells embedded in the basement membrane of blood microvessels, and uniquely positioned within the neurovascular unit between endothelial cells of capillaries [33, 34]. Previous studies in $\mathrm{A} \beta \mathrm{PP}$ transgenic mice have shown that pericyte loss can impede soluble $A \beta$ clearance and accelerate cerebral $\beta$ amyloidosis and CAA [35]. Further, our gene expression analysis in Fig. 4 showed that PDGFR $\beta$, a marker for pericytes, is reduced at 12 months of age in rTg-DI rats. Therefore, we evaluated microvascular pericytes in rTgDI rats at the different stages of the disease. In the wildtype rats, pericytes with extended processes were readily visualized along cerebral capillaries in all brain regions at 3 and 12 months of age (Fig. $5 \mathrm{a}-\mathrm{c}, \mathrm{d}-\mathrm{i}, \mathrm{m}$ ). In fact, the number of pericytes along cerebral capillaries actually significantly increased from 3 to 12 months in wildtype rats (Fig. 5o). At 3 months of age, with the onset of CAA, the morphology and numbers of capillary pericytes in rTg-DI rats were indistinguishable from wildtype rats (Fig. $5 \mathrm{~d}-\mathrm{f}, \mathrm{o}$ ). In contrast, at 12 months with extensive capillary amyloid deposition, pericytes were markedly reduced, degenerative, and lacked long extended process (Fig. $5 \mathrm{~d}-\mathrm{f}, \mathrm{n}$ ). Quantitation of pericyte coverage in cerebral capillaries in the cortex, hippocampus, and thalamus of rTg-DI rats revealed a highly significant reduction $(p<0.001)$ in all brain regions. These findings are consistent with the decreased expression of PDGFR $\beta$ at 12 months of age in this model of microvascular CAA (Fig. 4a).

\section{Increased numbers of caspase 3-positive cells are associated with severe microvascular amyloid deposition in rTg-DI rats}

Our findings above show that the extensive cerebral microvascular amyloid accumulation at 12 months in $\mathrm{rTg}$ DI rats is accompanied by robust neuroinflammation and loss of pericytes, recapitulating the pathological features of CAA type-1. In many human A $\beta$ PP transgenic mouse models that develop cerebral amyloid deposits and promote cell stress, elevated caspase 3 activation was observed [36-38]. Multiple lines of evidence indicate that caspase 3 activation is both necessary and sufficient to trigger apoptosis $[39,40]$. Since our above results clearly demonstrate that rTg-DI rats develop many signs of perivascular stress including strong neuroinflammation and loss of pericytes, we investigated if this also promoted caspase 3 activation in these brain regions. At 3 months of 


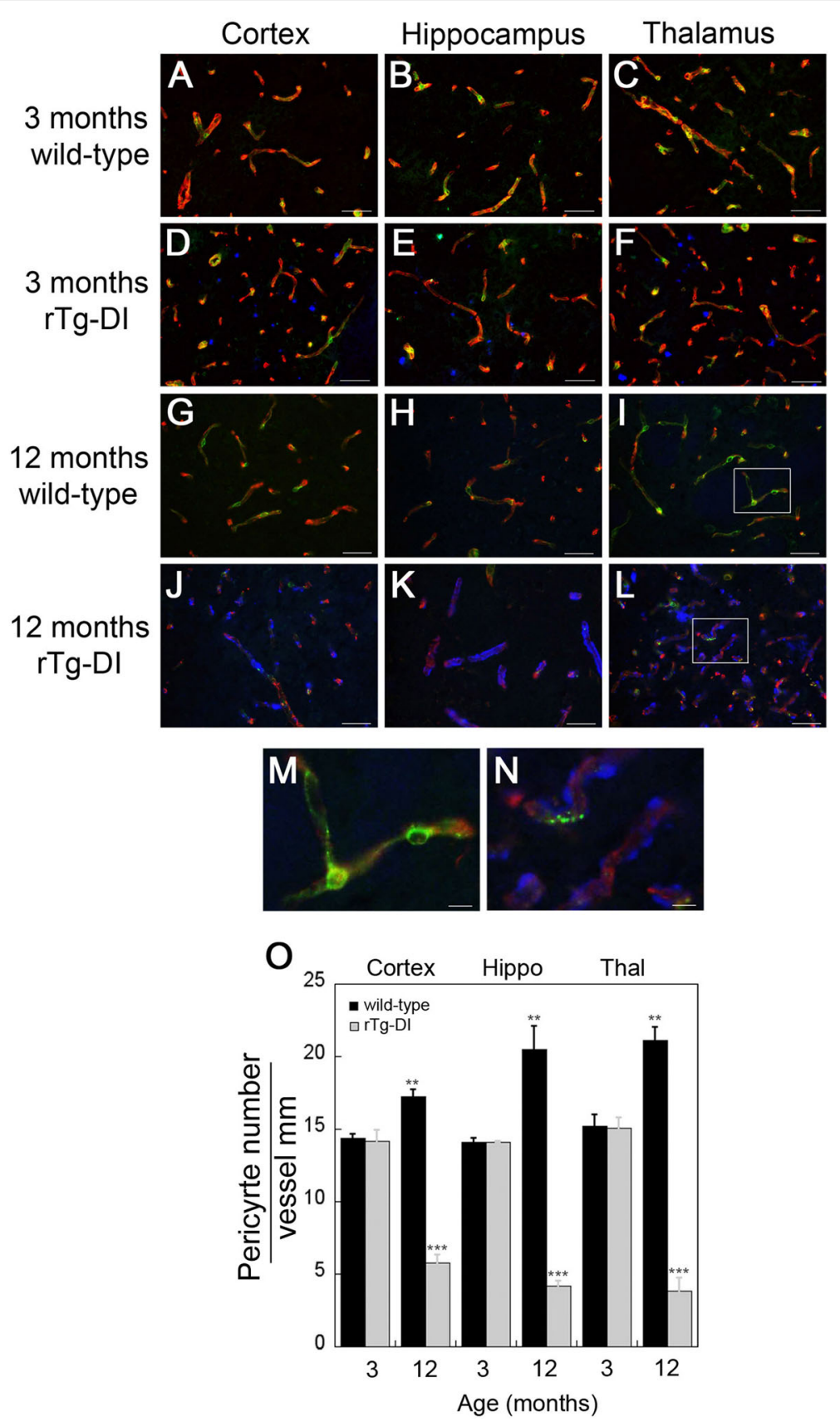

Fig. 5 Loss of perivascular pericytes in rTg-DI rats. a-I Brain sections from 3-month-old wild-type (a-c) and rTg-DI (d-f) rats and 12-month wildtype $(\mathbf{g}-\mathbf{i})$ and $\mathbf{r T g}-\mathrm{DI}(\mathbf{j}-\mathbf{I})$ rats were labeled with Amylo-Glo to detect fibrillar amyloid (blue), rabbit polyclonal antibody to collagen IV to detect cerebral microvessels (red), and goat polyclonal antibody to PDGFR $\beta$ for pericytes (green). Scale bars $=50 \mu \mathrm{m}$. $\mathbf{m}, \mathbf{n}$ Enlarged images of the highlighted regions of panels $\mathbf{i}$ and $\mathbf{I}$, respectively. Scale bars $=10 \mu \mathrm{m}$. o Quantitation of pericyte numbers from wild-type rats (black bars) and rTg-DI rats (gray bars) in different brain regions at 3 and 12 months of age. Data shown are mean \pm SD of $n=3-5$ rats per group. Pericyte numbers on capillaries increased in wild-type rats in all brain regions as they aged from 3 to 12 months. ${ }^{*} P<0.002$. In contrast, pericyte numbers markedly decreased in rTg-DI rats in all brain regions as they aged from 3 to 12 months. ${ }^{* * *} P<0.001$

age, the wild-type and rTg-DI rats with the absence of microvascular CAA, exhibited little evidence for caspase 3 activation (Fig. 6a-f, m). However, at 12 months of age in the presence of extensive CAA type- 1 , there was a dramatic increase in caspase 3-positive cells in rTg-DI rats
(Fig. 6j-l). Quantitative measures showed that there were highly significant increases of caspase 3-labeled cells in the cortex, hippocampus, and thalamus, with abundant microvascular CAA (Fig. 6m). To identify what cell types express caspase 3 in the 12 months old rTg-DI rats, 
double labeling for caspase 3 and cell-specific markers was performed. Surprisingly, these studies showed that the majority of caspase 3-positive cells were found to be astrocytes, with little, if any, involvement of neurons or microglia (Additional Fig. 4).

\section{Disruption of axonal integrity in rTg-DI with advanced CAA pathology}

We next explored if advancing CAA pathology and associated neuroinflammation had effects on surrounding neurons. Although we found no evidence of overt neuronal loss in the brains of rTg-DI rats up to 12 months of age (not shown), we then focused on axonal integrity. At 3 months of age, when cerebral microvascular amyloid deposition first appears in rTg-DI rats, axonal morphology appeared very similar to that of wild-type rats (Fig. $7 \mathrm{a}-\mathrm{f}$ ). However, as rTg-DI rats aged to 12 months and presented with extensive microvascular amyloid there were noted changes to axonal integrity (Fig. 7j-o). For example, swollen and fragmented axons were

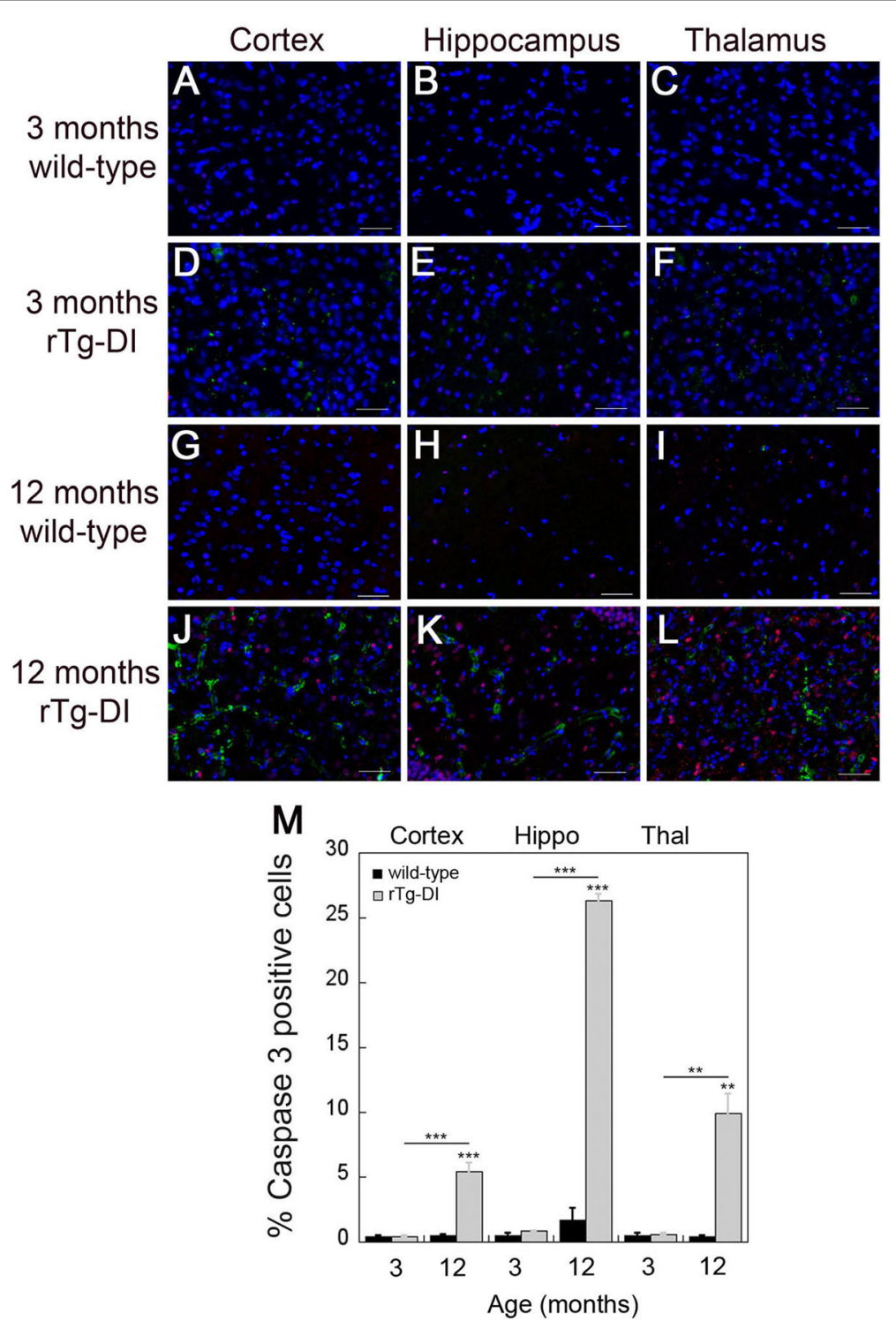

Fig. 6 Progressive accumulation of microvascular amyloid leads to increased numbers of activated caspase-3 positive cells in rTg-DI rats. a-f Representative images from 3-month-old wild-type $(\mathbf{a}-\mathbf{c})$ and $\mathbf{r T g}$-DI (d-f) rats and 12-month wild-type $(\mathbf{g}-\mathbf{i})$ and $\mathbf{r T g}$-DI (j-I) rats. Brain sections were stained with DAPI (blue), immunolabeled with a rabbit polyclonal antibody to active caspase 3 to identify apoptotic cells (red) and with mouse monoclonal antibody 66.1 to identify cerebral microvascular amyloid (green). Scale bars $=50 \mu \mathrm{m}$. $\mathbf{g}$ Quantitation of activated caspase 3 positive cells in wild-type rats (black bars) and rTg-DI rats (gray bars) in the cortex, hippocampus, and thalamus. Data shown are mean \pm SD of $n=5$ rats per group. ${ }^{*} P<0.01,{ }^{* *} P<0.001$. In wild-type rats, very few activated caspase 3 -positive cells were observed, but in $r T g-D I$ rat brains, markedly elevated numbers of activated caspase 3-positive cells were seen surrounding microvascular amyloid in all brain regions 
evident. In addition, many neuronal cell bodies were now labeled with the axonal marker showing a striking redistribution of this reactivity. These findings suggest that there are indeed marked impacts of advancing CAA pathology on neuronal integrity, which likely contribute to behavioral deficits in rTg-DI rats [16].

\section{Discussion}

CAA is a common cerebral small vessel disease of elderly people, a prominent feature of $\mathrm{AD}$, and a cause of VCID. However, our understanding of the etiology and downstream pathological consequences of cerebral vascular amyloid accumulation are still limited resulting in a lack of effective therapeutic treatments. Therefore, valid and consistent preclinical animal models to study the pathogenesis of CAA are paramount. Most previous animal studies on CAA involved the use of various human A $\beta P P$ transgenic mouse models that develop variable levels of CAA in the presence or absence of parenchymal amyloid pathology [41-44]. Recently, we reported the generation of a novel transgenic rat model (rTg-DI) that robustly develops capillary CAA type-1 [16]. We showed that rTg-DI rats express low amounts of human chimeric Dutch (E22Q)/Iowa
(D23N) familial CAA mutant $A \beta$ in the brain and develop early-onset and progressive microvascular CAA. As CAA progresses in rTg-DI rats, they develop consistent and numerous cerebral microbleeds that can readily be detected by magnetic resonance imaging. These findings suggest that rTg-DI rats have the potential to be a useful preclinical platform to study the pathogenesis of CAA type-1, to identify biomarkers for disease and to test therapeutic interventions. Indeed, we recently showed that decreasing levels of A 340 peptide in cerebrospinal fluid correlated with the progression of CAA in this model [22]. To better understand the utility of rTg-DI rats as a valid preclinical model, here we investigated the temporal development of several perivascular pathologies that are commonly observed with human CAA type-1. Our results show that with the progression of cerebral microvascular amyloid deposition, rTg-DI rats develop robust perivascular neuroinflammation, disruption and loss of capillary pericytes, increased numbers of caspase 3-positive cells, and disruption of axonal integrity. These findings indicate that rTg-DI rats faithfully recapitulate many of the pathological features of human CAA type- 1 and provide new insight into the pathogenesis of this condition.

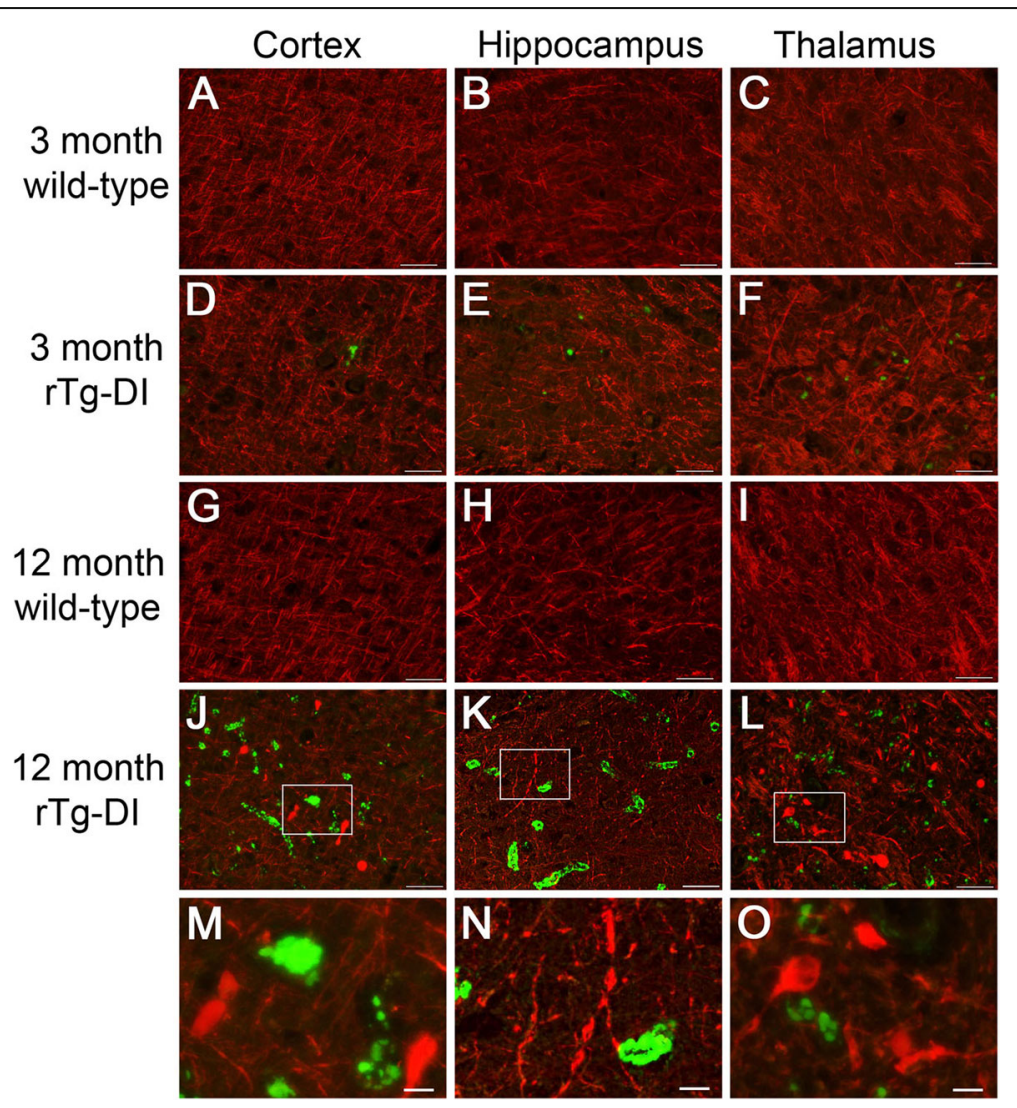

Fig. 7 Disrupted axonal integrity in rTg-DI rats. a-I Brain sections from 3-month-old wild-type (a-c) and rTg-DI (d-f) rats and 12-month wild-type $(\mathbf{g}-\mathbf{i})$ and $\mathbf{r T g}-\mathrm{DI}(\mathbf{j}-\mathbf{I})$ rats were labeled with thioflavin $\mathbf{S}$ to identify fibrillar amyloid (green) and pan axonal neurofilament marker (red). Scale bars $=50 \mu \mathrm{m} . \mathbf{m}, \mathbf{n}$, o Enlarged images of the highlighted regions of panels $\mathbf{j}, \mathbf{k}$, and $\mathbf{I}$, respectively. Scale bars $=10 \mu \mathrm{m}$. 
Neuroinflammation is recognized as a key component in the $\mathrm{AD}$ brain with marked increases and activation of glial cells surrounding parenchymal fibrillar amyloid plaques and expression of inflammatory mediators $[45,46]$. Likewise, in human CAA type-1, where vascular amyloid protrudes into the perivascular brain parenchyma, activated astrocytes and microglia accumulate in response to the amyloid [12, 47]. Similarly, in rTg-DI rats, we found that corresponding with the increasing accumulation of cerebral microvascular amyloid there is a dramatic elevation in the numbers of astrocytes and activated microglia (Figs. 2 and 3). These glial responses emerged at the onset of microvascular amyloid deposition $(\approx 3$ months of age) and increased as the disease progressed to an advanced stage (12 months of age). Interestingly, in late-stage disease the astrocytes around microvascular amyloid deposits exhibited enlarged cell bodies and retracted process suggesting a degenerative phenotype. Indeed, subsequent experiments showed a significant increase in caspase 3-positive cells that were largely identified as astrocytes. This suggests a distinct cytotoxic effect of the microvascular amyloid to astrocytes in this model. Similar to the increase in astrocytes, there was a pronounced elevation in numbers of microglia in the vicinity of microvascular amyloid deposits. Morphologically, these microglia presented in an activated state with a noticeable retraction of the extensive processes commonly seen in resting microglia of wildtype rats. To better understand the state of these activated microglia, labeling for MHCII OX-6 was performed to identify macrophagic microglia $[23,48]$. Clearly, there was a subset of microglia in rTg-DI rats that expressed both Iba- 1 and OX- 6 reactivity whereas many of the microglia only expressed Iba-1. This could suggest that dual-labeled microglia may derive from a different origin such as the periphery. However, recent studies suggest that microglia and macrophages can express similar or disparate markers depending on the environment where they exist (periphery vs brain) and the type of stimulus that leads to their activation [49]. Regarding this latter point, the activation stimulus here involves vascular amyloid and, in rTg-DI rats, involves familial Dutch/Iowa CAA mutant A $\beta$. Further specifying this pathogenic stimulus, we previously reported that in vascular amyloid, the $A \beta$ peptide adopts a distinct antiparallel fibril configuration $[16,50]$. Thus, the source and activation state of microglia can be unique for distinct disease states in the CNS and requires further investigation in this model [51]. In any case, regardless of the source, there is clearly a large microglial response to the microvascular amyloid in rTg-DI rats.

As mentioned above, reactive gliosis is a common response to a variety of neurodegenerative diseases including $\mathrm{AD}$, prion disease, Parkinson's disease, and multiple sclerosis $[52,53]$. In these diseases there exists a complex interaction between activated microglia and astrocytes mediated through pro- and anti-inflammatory cytokines/ chemokines that can lead to neuronal dysfunction. Our findings show that in response to microvascular amyloid, the striking glial activation promoted a distinct pattern of inflammatory marker expression in rTg-DI rats (Fig. 4). For example, elevated expression of certain proinflammatory cell markers such as GFAP, CD86, CD68, and TREM2 were noted that reflect the elevated numbers of astrocytes and microglia. Each of these glial markers were elevated at the onset of CAA and further increased with progression of microvascular amyloid accumulation. Increased expression of TREM2 in rTg-DI rats is of interest since mutations in this gene are associated with an increased risk for $\mathrm{AD}[54,55]$. Our findings may suggest a role for TREM2 in the pathogenesis of CAA as well. Increases in pro-inflammatory IL- $1 \beta$ and TNF- $\alpha$ were noted, yet other pro-inflammatory markers such as IL-6, IL-17, and IFN $\gamma$ did not increase with disease severity or were muted. On the other hand, TGF- $\beta 1$, more associated with anti-inflammatory pathways, was also expressed at high levels but IL-10, another anti-inflammatory cytokine, was not. Interestingly, we also found increased expression of alternative pathway complement component $\mathrm{C} 3$ in $\mathrm{rTg}$ DI rat brain, but not classical complement components $\mathrm{C} 1 \mathrm{q}$ and $\mathrm{C} 4$, which have been shown to have a tighter relationship with $A \beta$ plaque formation $[31,56]$. C1inhibitor, which regulates classical complement activation, was also increased in advanced disease. However, the lack of expression of classical complement components in $\mathrm{rTg}$ DI suggest that elevated C1-inhibitor may rather be in response to disrupted vessel integrity and microbleeds that are present only in the later stages of disease. There are indications for $\mathrm{C} 1$-inhibitor playing a role in reducing cerebral thrombo-inflammation [57]. Along these lines, MMP9 also showed increased expression at advanced stage of disease, and this is likely associated with the microbleed phenotype associated with CAA. These results indicate that the pathogenesis of CAA type- 1 in rTg-DI rats triggers neuroinflammation in the brain, but the gene expression pattern is unique, can be temporal, and differs from that of $A \beta$ plaque-related neuroinflammation. Along these lines, we recently reported that cerebral vascular $A \beta$ deposits have a unique, anti-parallel $\beta$-sheet fibril structure that is distinct from parenchymal plaque $A \beta$ fibrils that possess a parallel $\beta$-sheet fibril structure $[16,50]$. It is plausible that these distinct fibril structures promote distinct inflammatory signatures in the brain.

The spatial location of fibrillar amyloid deposits in CAA type-1 suggests direct interaction with pericytes, cells that regulate key neurovascular functions including blood-brain barrier formation and maintenance, clearance of toxic cellular byproducts, and regulating 
neuronal phenotype [58-60]. In rTg-DI rats, we found a dramatic reduction in the number of pericytes in the cortex, hippocampus, and thalamus at later stages of disease, consistent with the reduction in PDGFR $\beta$ expression. At the onset of CAA formation at 3 months, pericyte numbers and morphology were no different in rTg-DI rats compared with wild-type rats. In contrast, at 12 months there was considerable loss of extended processes along the length of the capillaries further underscoring the degenerative effects of accumulating amyloid on these cells. This is consistent with previous studies demonstrating that $A \beta$ is toxic to pericytes in primary cell culture [61]. Accordingly, we suggest that there is likely a negative feedback loop between pericyte loss and CAA progression in rTg-DI rats where the loss of pericytes diminishes proper neurovascular function and clearance of soluble $A \beta$ further promoting accumulation and deposition of $\mathrm{A} \beta$ on capillaries, which in turn amplifies amyloid-induced pericyte loss. Targeting this destructive interaction could have implications for developing strategies for early intervention in capillary CAA type- 1.

We found a marked increase in caspase 3-positive cells indicating a marker for cell stress and pro-apoptotic processes. This increase in caspase 3-positive cells was observed in late-stage disease, but not early-stage disease suggesting that it results from the continuing accumulation of microvascular amyloid and the chronic neuroinflammation associated with it. It was interesting that these stressed caspase 3-positive cells were largely determined to be astrocytes further implicating the impact of vascular amyloid accumulation on this cell population. Although we have not observed overt neuronal loss in this model at 12 months of age, this could become apparent in older animals. However, at around 12 months of age rTg-DI rats generally become moribund thus preventing further aging. Nevertheless, we did find that at later stage disease, when caspase 3-positive astrocytes are abundant, there were some profound effects on axonal morphology with fragmentation, swelling, and redistribution of axonal labeling. This implicates the increasing microvascular amyloid burden, chronic inflammation, and perivascular disruption in compromising neuronal integrity that likely underlies behavioral deficits in rTg-DI rats leading to VCID in this model.

\section{Conclusions}

The rTg-DI rat is a novel model of early-onset and progressive cerebral microvascular amyloid deposition that recapitulates many features of human CAA type-1. Our results show that there is a relationship between the onset and progressive accumulation of cerebral microvascular amyloid with the temporal development of neuroinflammation and perivascular cellular pathology.
Advanced stages of microvascular amyloid and neuroinflammation in rTg-DI rats is associated with pronounced pericyte loss in capillaries, degeneration of astrocytes, and disruption of neuronal axonal integrity. These findings underscore the utility of rTg-DI rats to serve as a useful preclinical platform to develop biomarkers and to test therapeutic strategies to intervene in the onset and progression of microvascular CAA and its role in VCID.

\section{Supplementary information}

Supplementary information accompanies this paper at https://doi.org/10 1186/s12974-020-01755-y.

Additional file 1 : Figure S1. Immunolabeling of astrocytes in 1 month old wild-type rats and rTg-DI rats prior to microvascular amyloid deposition. A-F: Brain sections from 1-month old wild-type (A-C) and rTg-DI (D-F) rats were labeled with Amylo-Glo to detect fibrillar amyloid (blue), rabbit polyclonal antibody to collagen IV to detect cerebral microvessels (red), and goat polyclonal antibody to GFAP to identify astrocytes (green). Scale bars $=10 \mu \mathrm{m}$. At this young age, in the absence of microvascular amyloid deposition astrocytes are morphologically indistinguishable between wild-type and rTg-DI rats.

Additional file $\mathbf{2}$ : Figure S2. Immunolabeling of microglia in 1 month old wild-type rats and rTg-DI rats prior to microvascular amyloid deposition. A-F: Brain sections from 1-month old wild-type (A-C) and rTg-DI (D-F) rats were labeled with Amylo-Glo to detect fibrillar amyloid (blue), rabbit polyclonal antibody to collagen IV to detect cerebral microvessels (red), and goat polyclonal antibody to Iba-1 to identify microglia (green). Scale bars $=10 \mu \mathrm{m}$. At this young age, in the absence of microvascular amyloid deposition microglia are morphologically indistinguishable between wild-type and rTg-DI rats with both exhibiting a resting phenotype.

Additional file $\mathbf{3}$ : Figure S3. Immunolabeling for macrophagic microglia in 3 and 12 month old wild-type rats and rTg-DI rats. A-L: Brain sections from 3-month old wild-type (A-C) and rTg-DI (D-F) rats and 12month wild-type $(\mathbf{G}-\mathbf{I})$ and $\mathbf{r T g}$-DI (K-L) rats were labeled with Amylo-Glo to detect fibrillar amyloid (blue), goat polyclonal antibody to Iba-1 as a marker for microglia (green) and mouse monoclonal antibody to OX6 as a marker for macrophages (red). Scale bars $=50 \mu \mathrm{m}$. In wild-type rats at both ages cells were solely labeled with Iba-1. In rTg-DI rats the majority of cells labeled solely with $\mathrm{lba}-1$ and a subset of cells were double labeled for the microglial marker Iba-1 and for the macrophagic microglial marker OX6. Few, if any, cells were labeled solely with OX6 antibody.

Additional file 4 : Figure S4. Double immunolabeling for caspase 3 and cell specific markers. A-C: Brain sections from 12 month old rTg-DI rats were immunolabeled for caspase 3 (red) and (A) NeuN to identify neurons (green), (B) GFAP to detect astrocytes (green) and (C) Iba-1 to identify microglia (green). Caspase 3 labeling most closely co-localized with astrocytes. Scale bars $=10 \mu \mathrm{m}$.

\section{Abbreviations}

AD: Alzheimer's disease; A $\beta$ : Amyloid beta protein; $A \beta P P$ : $A \beta$ precursor protein; $C 1$ inh: Complement component 1 esterase inhibitor; C1q: Complement component 1q; C3: Complement component 3; C4b: Complement component 4b; CAA: Cerebral amyloid angiopathy; CD68: cluster of differentiation 68; CD86: cluster of differentiation 68; DAB: Diaminobenzidene; GFAP: Glial fibrillar acidic protein; Iba-1: Ionized calcium-binding adapter molecule $1 ; \mathrm{ICH}$ : Intracerebral hemorrhage; IL10: Interleukin-10; IL-17: Interleukin-17; IL-1ß: Interleukin-1 $\beta$; IL-6: Interleukin-6; MHCII: Major histocompatibility complex II; MMP2: Matrix metalloproteinase 2; MMP9: Matrix metalloproteinase 9; OCT: Optimal cutting temperature medium; PDGFRB: Platelet-derived growth factor receptor $\beta ;$ PHF-1: Paired helical filament-1; rTg-DI: Transgenic rats that express Dutch/lowa mutant $A \beta$ in the brain; SD: Standard deviation; TGF $\beta 1$ : Transforming growth factor $\beta$; 
TNF-a: Tumor necrosis factor alpha; TREM2: Triggering receptor expressed on myeloid cells 2; VCID: Vascular cognitive impairment and dementia

\section{Acknowledgements}

We thank Ms. Judianne Davis and Ms. Aleksandra Stanisavljevic for the technical assistance.

\section{Authors' contributions}

XZ performed the study design, tissue collection, histological analyses, stereology and qPCR; analyzed and interpreted the data; and was a major contributor in writing the manuscript. JH assisted with the histological analyses and stereology; analyzed and interpreted the data; and was a contributor in writing the manuscript. JS assisted with the study design, tissue collection, and qPCR and analyzed and interpreted the data. FX assisted with tissue collection and histological analyses. WN designed the experiments, interpreted data, and was a major contributor in writing the manuscript. All authors read and approved the final manuscript.

\section{Funding}

This work was supported by the NIH grants RO1NS094201 and R21NS091969.

\section{Availability of data and materials}

The datasets used and/or analyzed during the current study are available from the corresponding author on reasonable request.

\section{Ethics approval and consent to participate}

All experiments were conducted in accordance with the United States Public Health Service's Policy on Humane Care and Use of Laboratory Animals and approved by the University of Rhode Island Institutional Animal Care and Use Committee (project \#AN1718-008; approval dates 12/11/2017-12/10/ 2020)

\section{Consent for publication}

Not applicable.

\section{Competing interests}

The authors declare that they have no competing interests.

\section{Author details}

${ }^{1}$ Department of Biomedical and Pharmaceutical Sciences, College of Pharmacy, George \& Anne Ryan Institute for Neuroscience, University of Rhode Island, 130 Flagg Road, Kingston, RI 02881, USA. ${ }^{2}$ Present Address: Cold Spring Harbor Laboratory, 1 Bungtown Road, Cold Spring Harbor, NY 11724, USA. ${ }^{3}$ Present Address: New York Medical College, 40 Sunshine Cottage Road, Valhalla, NY 10595, USA

Received: 4 November 2019 Accepted: 21 February 2020

Published online: 04 March 2020

\section{References}

1. Attems J, Jellinger $K$, Thal DR, Van Nostrand W. Review: sporadic cerebral amyloid angiopathy. Neuropathol Appl Neurobiol. 2011;37:75-93.

2. Rensink AA, de Waal RM, Kremer B, Verbeek MM. Pathogenesis of cerebral amyloid angiopathy. Brain Res Brain Res Rev. 2003:43:207-23.

3. Arvanitakis Z, Leurgans SE, Wang Z, Wilson RS, Bennett DA, Schneider JA. Cerebral amyloid angiopathy pathology and cognitive domains in older persons. Ann Neurol. 2011;69:320-7.

4. Viswanathan A, Greenberg SM. Cerebral amyloid angiopathy in the elderly. Ann Neurol. 2011;70:871-80.

5. Levy E, Carman MD, Fernandez-Madrid IJ, Power MD, Lieberburg I, van Duinen SG, Bots GT, Luyendijk W, Frangione B. Mutation of the Alzheimer's disease amyloid gene in hereditary cerebral hemorrhage, Dutch type. Science. 1990:248:1124-6.

6. Van Broeckhoven C, Haan J, Bakker E, Hardy JA, Van Hul W, Wehnert A, Vegter-Van der Vlis M, Roos RA. Amyloid beta protein precursor gene and hereditary cerebral hemorrhage with amyloidosis (Dutch). Science. 1990;248: $1120-2$.

7. Grabowski TJ, Cho HS, Vonsattel JP, Rebeck GW, Greenberg SM. Novel amyloid precursor protein mutation in an lowa family with dementia and severe cerebral amyloid angiopathy. Ann Neurol. 2001;49:697-705.
8. Boyle PA, Yu L, Nag S, Leurgans S, Wilson RS, Bennett DA, Schneider JA. Cerebral amyloid angiopathy and cognitive outcomes in community-based older persons. Neurology. 2015;85:1930-6.

9. Greenberg SM, Vernooij MW, Cordonnier C, Viswanathan A, Al-Shahi Salman R, Warach S, Launer LJ, Van Buchem MA, Breteler MM, Microbleed Study G. Cerebral microbleeds: a guide to detection and interpretation. Lancet Neurol. 2009;8:165-74.

10. Thal DR, Ghebremedhin E, Rub U, Yamaguchi H, Del Tredici K, Braak H. Two types of sporadic cerebral amyloid angiopathy. J Neuropathol Exp Neurol. 2002:61:282-93.

11. Eikelenboom P, Veerhuis R, Familian A, Hoozemans JJ, van Gool WA, Rozemuller AJ. Neuroinflammation in plaque and vascular beta-amyloid disorders: clinical and therapeutic implications. Neurodegener Dis. 2008;5: 190-3.

12. Richard E, Carrano A, Hoozemans JJ, van Horssen J, van Haastert ES, Eurelings LS, de Vries HE, Thal DR, Eikelenboom P, van Gool WA, Rozemuller AJ. Characteristics of dyshoric capillary cerebral amyloid angiopathy. J Neuropathol Exp Neurol. 2010;69:1158-67.

13. Attems J, Jellinger KA. Only cerebral capillary amyloid angiopathy correlates with Alzheimer pathology--a pilot study. Acta Neuropathol. 2004:107:83-90.

14. Bailey TL, Rivara CB, Rocher AB, Hof PR. The nature and effects of cortical microvascular pathology in aging and Alzheimer's disease. Neurol Res. 2004; 26:573-8

15. Thal DR, Ghebremedhin E, Orantes M, Wiestler OD. Vascular pathology in Alzheimer disease: correlation of cerebral amyloid angiopathy and arteriosclerosis/lipohyalinosis with cognitive decline. J Neuropathol Exp Neurol. 2003;62:1287-301.

16. Davis J, Xu F, Hatfield J, Lee H, Hoos MD, Popescu D, Crooks E, Kim R, Smith $\mathrm{SO}$, Robinson JK, et al. A novel transgenic rat model of robust cerebral microvascular amyloid with prominent vasculopathy. Am J Pathol. 2018;188: 2877-89.

17. Long JM, Kalehua AN, Muth NJ, Hengemihle JM, Jucker M, Calhoun ME, Ingram DK, Mouton PR. Stereological estimation of total microglia number in mouse hippocampus. J Neurosci Methods. 1998;84:101-8.

18. Deane R, Du Yan S, Submamaryan RK, LaRue B, Jovanovic S, Hogg E, Welch D, Manness L, Lin C, Yu J, et al. RAGE mediates amyloid-beta peptide transport across the blood-brain barrier and accumulation in brain. Nat Med. 2003;9:907-13.

19. Batarseh YS, Duong QV, Mousa YM, Al Rihani SB, Elfakhri K, Kaddoumi A. Amyloid-beta and astrocytes interplay in amyloid-beta related disorders. Int J Mol Sci. 2016:17:338.

20. Zabel M, Schrag M, Crofton A, Tung S, Beaufond P, Van Ornam J, Dininni A, Vinters HV, Coppola G, Kirsch WM. A shift in microglial beta-amyloid binding in Alzheimer's disease is associated with cerebral amyloid angiopathy. Brain Pathol. 2013;23:390-401.

21. Kettenmann H, Hanisch UK, Noda M, Verkhratsky A. Physiology of microglia. Physiol Rev. 2011;91:461-553.

22. Zhu X, Xu F, Hoos MD, Lee H, Benveniste H, Nostrand WEV. Reduced levels of cerebrospinal fluid/plasma $A \beta 40$ as an early biomarker for cerebral amyloid angiopathy in RTg-DI rats. Int J Mol Sci. 2020;21.

23. Patro N, Nagayach A, Patro IK. Iba1 expressing microglia in the dorsal root ganglia become activated following peripheral nerve injury in rats. Indian J Exp Biol. 2010;48:110-6.

24. Kinnecom C, Lev MH, Wendell L, Smith EE, Rosand J, Frosch MP, Greenberg SM. Course of cerebral amyloid angiopathy-related inflammation. Neurology. 2007;68:1411-6.

25. Miao J, Xu F, Davis J, Otte-Holler I, Verbeek MM, Van Nostrand WE. Cerebral microvascular amyloid beta protein deposition induces vascular degeneration and neuroinflammation in transgenic mice expressing human vasculotropic mutant amyloid beta precursor protein. Am J Pathol. 2005; 167:505-15.

26. Colton CA, Mott RT, Sharpe H, Xu Q, Van Nostrand WE, Vitek MP. Expression profiles for macrophage alternative activation genes in $A D$ and in mouse models of AD. J Neuroinflammation. 2006;3:27.

27. Cherry JD, Olschowka JA, O'Banion MK. Neuroinflammation and M2 microglia: the good, the bad, and the inflamed. J Neuroinflammation. 2014;11:98.

28. Mills CD, Kincaid K, Alt JM, Heilman MJ, Hill AM. M-1/M-2 macrophages and the Th1/Th2 paradigm. J Immunol. 2000;164:6166-73.

29. Boche D, Perry VH, Nicoll JA. Review: activation patterns of microglia and their identification in the human brain. Neuropathol Appl Neurobiol. 2013;39:3-18. 
30. Kawanokuchi J, Shimizu K, Nitta A, Yamada K, Mizuno T, Takeuchi H, Suzumura A. Production and functions of IL-17 in microglia. J Neuroimmunol. 2008;194:54-61.

31. Crehan H, Hardy J, Pocock J. Microglia, Alzheimer's disease, and complement. Int J Alzheimers Dis. 2012;2012:983640.

32. Zhao $B Q$, Tejima $E$, Lo EH. Neurovascular proteases in brain injury, hemorrhage and remodeling after stroke. Stroke. 2007;38:748-52.

33. Armulik A, Genove G, Betsholtz C. Pericytes: developmental, physiological, and pathological perspectives, problems, and promises. Dev Cell. 2011;21: 193-215.

34. Zlokovic BV. The blood-brain barrier in health and chronic neurodegenerative disorders. Neuron. 2008;57:178-201.

35. Sagare AP, Bell RD, Zhao Z, Ma Q, Winkler EA, Ramanathan A, Zlokovic BV. Pericyte loss influences Alzheimer-like neurodegeneration in mice. Nat Commun. 2013:4:2932

36. Hwang DY, Chae KR, Kang TS, Hwang JH, Lim CH, Kang HK, Goo JS, Lee MR, Lim HJ, Min SH, et al. Alterations in behavior, amyloid beta-42, caspase-3, and Cox-2 in mutant PS2 transgenic mouse model of Alzheimer's disease. FASEB J. 2002;16:805-13.

37. D'Amelio M, Cavallucci V, Middei S, Marchetti C, Pacioni S, Ferri A, Diamantini A, De Zio D, Carrara P, Battistini L, et al. Caspase-3 triggers early synaptic dysfunction in a mouse model of Alzheimer's disease. Nat Neurosci. 2011;14:69-76.

38. Yang DS, Kumar A, Stavrides P, Peterson J, Peterhoff CM, Pawlik M, Levy E, Cataldo AM, Nixon RA. Neuronal apoptosis and autophagy cross talk in aging PS/APP mice, a model of Alzheimer's disease. Am J Pathol. 2008;173:665-81.

39. Nagata S. Apoptosis by death factor. Cell. 1997;88:355-65.

40. Thornberry NA, Lazebnik Y. Caspases: enemies within. Science. 1998;281: 1312-6.

41. Calhoun ME, Burgermeister P, Phinney AL, Stalder M, Tolnay M, Wiederhold KH, Abramowski D, Sturchler-Pierrat C, Sommer B, Staufenbiel M, Jucker M. Neuronal overexpression of mutant amyloid precursor protein results in prominent deposition of cerebrovascular amyloid. Proc Natl Acad Sci U S A. 1999:96:14088-93.

42. Davis J, Xu F, Deane R, Romanov G, Previti ML, Zeigler K, Zlokovic BV, Van Nostrand WE. Early-onset and robust cerebral microvascular accumulation of amyloid beta-protein in transgenic mice expressing low levels of a vasculotropic Dutch/lowa mutant form of amyloid beta-protein precursor. J Biol Chem. 2004;279:20296-306.

43. Herzig MC, Winkler DT, Burgermeister P, Pfeifer M, Kohler E, Schmidt SD, Danner S, Abramowski D, Sturchler-Pierrat C, Burki K, et al. A $\beta$ is targeted to the vasculature in a mouse model of hereditary cerebral hemorrhage with amyloidosis. Nat Neurosci. 2004;7:954-60.

44. Oakley H, Cole SL, Logan S, Maus E, Shao P, Craft J, Guillozet-Bongaarts A, Ohno M, Disterhoft J, Van Eldik L, et al. Intraneuronal beta-amyloid aggregates, neurodegeneration, and neuron loss in transgenic mice with five familial Alzheimer's disease mutations: potential factors in amyloid plaque formation. J Neurosci. 2006;26:10129-40.

45. Heneka MT, Carson MJ, El Khoury J, Landreth GE, Brosseron F, Feinstein DL, Jacobs AH, Wyss-Coray T, Vitorica J, Ransohoff RM, et al. Neuroinflammation in Alzheimer's disease. Lancet Neurol. 2015;14:388-405.

46. Medeiros R, LaFerla FM. Astrocytes: conductors of the Alzheimer disease neuroinflammatory symphony. Exp Neurol. 2013;239:133-8.

47. Van Nostrand WE, XU F, Rozemuller AJ, Colton CA. Enhanced capillary amyloid angiopathy-associated pathology in Tg-SwDI mice with deleted nitric oxide synthase 2. Stroke. 2010;41:S135-8.

48. Marshall SA, McClain JA, Kelso ML, Hopkins DM, Pauly JR, Nixon K. Microglial activation is not equivalent to neuroinflammation in alcohol-induced neurodegeneration: the importance of microglia phenotype. Neurobiol Dis. 2013;54:239-51.

49. Grassivaro F, Menon R, Acquaviva M, Ottoboni L, Ruffini F, Bergamaschi A, Muzio L, Farina C, Martino G. Convergence between microglia and peripheral macrophages phenotype during development and neuroinflammation. J Neurosci. 2020;40:784-95.

50. Xu F, Fu Z, Dass S, Kotarba AE, Davis J, Smith SO, Van Nostrand WE. Cerebral vascular amyloid seeds drive amyloid beta-protein fibril assembly with a distinct anti-parallel structure. Nat Commun. 2016;7:13527.

51. Varvel NH, Grathwohl SA, Baumann F, Liebig C, Bosch A, Brawek B, Thal DR, Charo IF, Heppner FL, Aguzzi A, et al. Microglial repopulation model reveals a robust homeostatic process for replacing CNS myeloid cells. Proc Natl Acad Sci U S A. 2012;109:18150-5.
52. Burda JE, Sofroniew MV. Reactive gliosis and the multicellular response to CNS damage and disease. Neuron. 2014;81:229-48.

53. Pekny M, Pekna M. Reactive gliosis in the pathogenesis of CNS diseases. Biochim Biophys Acta. 1862;2016:483-91.

54. Jonsson T, Stefansson H, Steinberg S, Jonsdottir I, Jonsson PV, Snaedal J, Bjornsson S, Huttenlocher J, Levey Al, Lah JJ, et al. Variant of TREM2 associated with the risk of Alzheimer's disease. N Engl J Med. 2013;368:107-16.

55. Guerreiro R, Wojtas A, Bras J, Carrasquillo M, Rogaeva E, Majounie E, Cruchaga C, Sassi C, Kauwe JS, Younkin S, et al. TREM2 variants in Alzheimer's disease. N Engl J Med. 2013;368:117-27.

56. Emmerling MR, Watson MD, Raby CA, Spiegel K. The role of complement in Alzheimer's disease pathology. Biochim Biophys Acta. 2000;1502:158-71.

57. Albert-Weissenberger C, Mend S, Schuhmann MK, Salur I, Gob E, Langhauser F, Hopp S, Hennig N, Meuth SG, Nolte MW, et al. C1-inhibitor protects from focal brain trauma in a cortical cryolesion mice model by reducing thrombo-inflammation. Front Cell Neurosci. 2014;8:269.

58. Armulik A, Genove G, Mae M, Nisancioglu MH, Wallgard E, Niaudet C, He L, Norlin J, Lindblom P, Strittmatter K, et al. Pericytes regulate the blood-brain barrier. Nature. 2010;468:557-61.

59. Daneman $R$, Zhou $L$, Kebede AA, Barres BA. Pericytes are required for bloodbrain barrier integrity during embryogenesis. Nature. 2010;468:562-6.

60. Bell RD, Winkler EA, Sagare AP, Singh I, LaRue B, Deane R, Zlokovic BV Pericytes control key neurovascular functions and neuronal phenotype in the adult brain and during brain aging. Neuron. 2010;68:409-27.

61. Verbeek MM, de Waal RM, Schipper JJ, Van Nostrand WE. Rapid degeneration of cultured human brain pericytes by amyloid beta protein. J Neurochem. 1997;68:1135-41.

\section{Publisher's Note}

Springer Nature remains neutral with regard to jurisdictional claims in published maps and institutional affiliations.
Ready to submit your research? Choose BMC and benefit from:

- fast, convenient online submission

- thorough peer review by experienced researchers in your field

- rapid publication on acceptance

- support for research data, including large and complex data types

- gold Open Access which fosters wider collaboration and increased citations

- maximum visibility for your research: over $100 \mathrm{M}$ website views per year

At BMC, research is always in progress.

Learn more biomedcentral.com/submissions 